\title{
A semi-analytic explicit integrator for stochastic differential equations driven by multidimensional linear multiplicative noise
}

Hugo de la Cruz ${ }^{1}$

Escola de Matemática Aplicada, Fundação Getulio Vargas-FGV/EMAp, RJ

\begin{abstract}
Many important stochastic differential equations (SDE) used for modeling noisy dynamical systems are driven by multidimensional linear multiplicative noise. In this work we introduce an explicit, stable and easily implementable numerical integrator specially devised for such a class of stochastic systems. The pathwise convergence -under non-standard assumption on the coefficients of the SDE- is studied. Remarkably, we show that even though the proposed method is explicit, it is unconditionally MS-stable and consequently much more efficient than methods commonly used in the literature to stably integrate this kind of equations. Some questions related to the computational implementation of the method are also discussed.
\end{abstract}

Keywords. stochastic differential equations, random differential equations, numerical approximation, stability, convergence, local linearization method

\section{Introduction}

Dynamical systems are often subject to noisy fluctuations, which may have a high impact on their evolution. When the dynamics of such noisy systems has to be studied, deterministic equations are obviously not suitable [1]. An usual and, typically, appropriate way of mathematically modelling these systems in throught stochastic differential equations (SDEs) of the form

$$
d x(t)=a(x(t)) d t+\sum_{j=1}^{m} b_{j}(x(t)) d w_{t}^{j},
$$

where $\left(w_{t}^{1}, \ldots, w_{t}^{m}\right)_{t \in \mathbb{R}^{+}}$denotes an $m$-dimensional standard Wiener process, defined by the properties: $w_{t}^{j}$ is Gaussian distributed $\left(w_{t}^{j} \sim N(0, t)\right)$, increments $w_{t}^{j}-w_{s}^{j} \sim N(0, t-s)$ are independent on non overlapping subintervals and $w_{0}^{j}=0$ w.p.1. This equation, in fact, should be interpreted in the integral form

$$
x(t)=x\left(t_{0}\right)+\int_{t_{0}}^{t} a(x(s)) d s+\sum_{j=1}^{m} \int_{t_{0}}^{t} b_{j}(x(s)) d w_{s}^{j},
$$

\footnotetext{
${ }^{1}$ hugo.delacruz@fgv.br
} 
where the first integral is a deterministic Riemann integral and the last $m$ integrals (called Itô stochastic integrals) are defined as the mean-square limit

$$
\int_{t_{0}}^{t} b_{j}(x(s)) d w_{s}^{j}:=(m . s) \lim _{N \rightarrow \infty} \sum_{k=0}^{N-1} b_{j}\left(x\left(t_{k}\right)\right)\left(w_{k+1}^{j}-w_{k}^{j}\right),
$$

taken over partitions $t_{0}<t_{1}<\cdots<t_{N}=t$ of the interval $\left[t_{0} T\right]$, with $\max _{k=0, \ldots, N-1}\left(t_{k+1}-\right.$ $\left.t_{k}\right) \rightarrow 0$ as $N \rightarrow \infty$. The functions $a$ and $b_{j}$ are called the drift and diffusion, respectively. An important class of widely used SDEs for modeling nonlinear systems are defined by quite complicated nonlinear drift component and linear multiplicative noise diffusion terms (i.e., $\left.b_{j}=\sigma_{j}(t) x(t)\right)$. Hence, they are of the form

$$
x(t)=x\left(t_{0}\right)+\int_{t_{0}}^{t} a(x(s)) d s+\sum_{j=1}^{m} \int_{t_{0}}^{t} \sigma_{j}(t) x(t) d w_{s}^{j} .
$$

For example, the well-known stochastic Ginzburg-Landau equation [6]

$$
d x=\left(\alpha x-\lambda x^{3}\right) d t+\sigma x d w_{t}, \quad \alpha, \lambda, \sigma \in \mathbb{R}^{+},
$$

and the stochastic Verhulst equation, for a population with competition between individuals [7]

$$
d x=\left(\left(\eta+\frac{1}{2} \sigma^{2}\right) x-\lambda x^{2}\right) d t+\sigma x d w_{t}, \quad \eta, \lambda, \sigma \in \mathbb{R}^{+},
$$

belong to this class of SDEs.

Just as in the deterministic case, closed-form expressions for the solution of SDEs, in particular for (2), are often unobtainable, and so the construction of approximation methods for the treatment and simulation of (2) is an important need. There exists a variety of important issues in designing practical numerical schemes. An appropriate tradeoff among convergence, stability, and computational efficiency is desirable but difficult to achieve. In particular, in the stochastic scenario, unconditional stability of, efficient, numerical schemes is perhaps the more important property, and that is why many efforts have been directed in this direction. However, most of the proposed methods in the literature that have good stability properties are of an implicit nature which is a major drawback from a practical point of view. Therefore, the construction of explicit and stable methods is of great interest when designing numerical methods for SDEs.

On the other hand, to guarantee convergence of many numerical integrators, strong assumptions must be assumed on the drift and diffusion of the equation (see for instance [7]). For instance, typical assumptions for the convergence of Itô-Taylor schemes assume global Lipschitz conditions or uniformly bounded partial derivatives of the coefficient functions $a, b_{j}$. This is very restrictive, since these assumptions are not satisfied for many SDEs in important applications such as the stochastic Ginzburg-Landau equation (3) and the Verhulst equation (4), just to mention a few examples. 
The aim of this paper is to derive an explicit and stable integrator for the pathwise approximation of the SDE (2). For this, in contrast with standard approaches for devising numerical methods for SDEs (see e.g., [7] and references therein) we will exploit the special structure of (2) which allows the equation to be transformed to a suitable Random Differential Equation, which can in turn be successfully integrated in a stable way -by using an exponential-based local linearization technique- even when the drift coefficient in (2) does not satisfy the aforementioned standard assumptions on the drift coefficient. We note that, in comparison with the method obtained in [3], the scheme proposed in this work is simpler and easier to implement when applied to the underlined equation (2).

The paper is organized as follows. In the next section some preliminary results are presented. Section 3 presents the deduction of the proposed method and the convergence and stability is considered. In section 4 are given details on the effective implementation of the proposed method.

\section{Some preliminaries}

\subsection{Random Differential Equations and LL approximation}

Let $(\Omega, \mathcal{F}, P)$ be a complete probability space, and $\left(\mathcal{F}_{t}\right)_{t>0}$ be an increasing right continuous family of complete sub $\sigma$-algebras of $\mathcal{F}$ and $P$ a probability measure. Let $\xi_{t}(\omega)=\left(\xi_{t}^{1}(\omega), \ldots, \xi_{t}^{k}(\omega)\right):\left[t_{0}, T\right] \times \Omega \longrightarrow \mathbb{R}^{k}$ an $k$-dimensional continuous stochastic processes. A random differential equation (RDE) (see [4])

$$
\begin{aligned}
& z^{\prime}(t)=f\left(z(t), \xi_{t}(\omega)\right), \quad t \in\left[t_{0}, T\right], \\
& z\left(t_{0}\right)=z_{0},
\end{aligned}
$$

is essentially an non-autonomous ODE for almost all realization $\omega \in \Omega$. For the sake of simplicity, from now on we will work with $\xi_{t}$ i.e., without an explicit reference to the realization $\omega$.

\subsubsection{LL method for RDEs}

Let $(\tau)_{h}: t_{0}<t_{1}<\ldots<t_{N}=T$ be a partition of the time interval $\left[t_{0}, T\right]$ with, for simplicity, equidistant stepsize $h<1$, i.e., defined as a sequence of times such that $t_{n}=t_{0}+n h$, for $n=0,1, \ldots, N$. Starting from the initial value $z_{0}$, the approximations $\left\{z_{i}\right\}$ to $\left\{z\left(t_{i}\right)\right\},(i=1,2, \ldots, N)$ are obtained recursively as follows.

$$
z_{n+1}=z_{n}+\left[\begin{array}{lll}
1 & 0 & 0
\end{array}\right] \exp (h M)\left[\begin{array}{lll}
0 & 0 & 1
\end{array}\right]^{\top},
$$

with

$$
M=\left[\begin{array}{ccc}
\frac{\partial f}{\partial z}\left(z_{n}, \xi_{t_{n}}\right) & \frac{\partial f}{\partial \xi}\left(z_{n}, \xi_{t_{n}}\right) \cdot \frac{\Delta \xi_{t_{n}}}{h} & f\left(z_{n}, \xi_{t_{n}}\right) \\
0 & 0 & 1 \\
0 & 0 & 0
\end{array}\right]
$$

Note that $\frac{\partial f}{\partial \xi} \cdot \frac{\Delta \xi_{t_{n}}}{h}=\frac{1}{h}\left(\sum_{j=1}^{k} \frac{\partial f}{\partial \xi^{j}} \cdot \Delta \xi_{t_{n}}^{j}\right), \Delta \xi_{t_{n}}^{j}=\xi_{t_{n+1}}^{j}-\xi_{t_{n}}^{j}$. We refer to [2] for details about how this method can be obtained. 


\subsection{A simple result from stochastic calculus}

The following result will be used later in the deduction of the proposed method (see e.g., [1]).

Lemma 2.1. The solution to the equation

$$
\begin{aligned}
d \phi(t) & =\sum_{j=1}^{m} \sigma_{j}(t) \phi(t) d w_{t}^{j}, \\
\phi\left(t_{0}\right) & =1
\end{aligned}
$$

is

$$
\phi(t)=\exp \left(-\frac{1}{2} \int_{t_{0}}^{t}\left(\sum_{j=1}^{m} \sigma_{j}^{2}(s)\right) d s+\sum_{j=1}^{m} \int_{t_{0}}^{t} \sigma_{j}(s) d w_{s}^{j}\right) .
$$

Also, by Itô-formula to $\phi^{-1}(t)$ (note that $\phi(t)>0$, hence $\exists \phi^{-1}(t)$ ) we have

$$
d \phi^{-1}(t)=\left(\sum_{j=1}^{m} \sigma_{j}^{2}(t)\right) \phi^{-1}(t) d t-\sum_{j=1}^{m} \sigma_{j}(t) \phi^{-1}(t) d w_{t}^{j} .
$$

\section{The proposed integrator}

Let $z(t)=\phi^{-1}(t) x(t)$, where $\phi^{-1}(t)$ and $x(t)$ satisfy the equations (9) and (2), we calculate the stochastic differential of $z(t)$. By Itô-formula and the stochastic product rule

$$
d z(t)=\phi^{-1}(t) d x(t)+d \phi^{-1}(t) x(t)+d \phi^{-1}(t) d x(t)=\phi^{-1}(t) a(x(s)) d t
$$

thus, $z(t)$ satisfies the following RDE

$$
\begin{aligned}
& z^{\prime}(t)=\phi^{-1}(t) a(\phi(t) z(s)), \quad t \in\left[t_{0}, T\right], \\
& z\left(t_{0}\right)=x_{0},
\end{aligned}
$$

Then, based on the partition $(\tau)_{h}$, the numerical integrator for the original SDE (1) is defined by

$$
x_{k}=\phi\left(t_{k}\right) z_{k}, \quad k=1, \ldots, N
$$

where $z_{k}$ is the LL approximation (6) to (10), i.e., for $f\left(z(t), \xi_{t}(\omega)\right)=\xi_{t}^{1} a\left(\xi_{t}^{2} z(s)\right)$ in (5), with $\xi_{t}^{1}=\phi^{-1}(t), \xi_{t}^{2}=\phi(t)$.

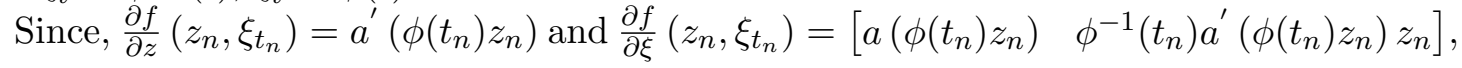

$$
z_{n+1}=z_{n}+\left[\begin{array}{lll}
1 & 0 & 0
\end{array}\right] \exp (h M)\left[\begin{array}{lll}
0 & 0 & 1
\end{array}\right]^{\top},
$$

with 
$M=\left[\begin{array}{ccc}a^{\prime}\left(\phi\left(t_{n}\right) z_{n}\right) & a\left(\phi\left(t_{n}\right) z_{n}\right) \cdot \frac{\Delta \phi_{t_{n}}^{-1}}{h}+\phi^{-1}\left(t_{n}\right) a^{\prime}\left(\phi\left(t_{n}\right) z_{n}\right) z_{n} \cdot \frac{\Delta \phi_{t_{n}}}{h} & \phi^{-1}\left(t_{n}\right) a\left(\phi\left(t_{n}\right) z_{n}\right) \\ 0 & 0 & 0\end{array}\right]$.

To compute $\phi\left(t_{n}\right)$ and $\phi\left(t_{n+1}\right)$ we make use of (8). The involved integrals in (8), namely $\int_{t_{0}}^{t_{n}} \sigma_{j}^{2}(s) d s$ and $\int_{t_{0}}^{t_{n}} \sigma_{j}(s) d w_{s}^{j}$, need to be accurately computed (a second order approximation is enough for our purpose, as will be seen later in the section). First, we apply a Trapezoidal quadrature in the subintervals of the partition $(\tau)_{h}$ to obtain the approximation

$$
\int_{t_{k}}^{t_{k+1}} \sigma_{j}^{2}(s) d s \approx I_{j k}=\frac{h}{2}\left(\sigma_{j}^{2}\left(t_{k}\right)+\sigma_{j}^{2}\left(t_{k+1}\right)\right), \quad k=0, \ldots, N-1,
$$

then we have the order 2 approximation

$$
\int_{t_{0}}^{t_{n}} \sigma_{j}^{2}(s) d s \approx \sum_{k=0}^{n-1} I_{j k}
$$

To accurately simulate the stochastic integral $\int_{t_{0}}^{t_{n}} \sigma_{j}(s) d w_{s}^{j}$, we first generate $\int_{t_{k}}^{t_{k+1}} \sigma_{j}(s) d w_{s}^{j}$ from a Gaussian distribution (by using the Itô-isometry property [1])

$$
\int_{t_{k}}^{t_{k+1}} \sigma_{j}(s) d w_{s}^{j} \sim N\left(0, \int_{t_{k}}^{t_{k+1}} \sigma_{j}^{2}(s) d s\right) \approx I_{j k} \cdot \gamma_{j k}, \quad \text { where } \gamma_{j k} \text { is } i . i . d \sim N(0,1) .
$$

Then

$$
\int_{t_{0}}^{t_{n}} \sigma_{j}(s) d w_{s}^{j} \approx \sum_{k=0}^{n-1}\left(I_{j k} \cdot \gamma_{j k}\right)
$$

Hence, from (8)

$$
\phi\left(t_{n}\right) \approx \hat{\phi}\left(t_{n}\right)=\exp \left(\sum_{j=1}^{m} \sum_{k=0}^{n-1} I_{j k}\left(-\frac{1}{2}+\gamma_{j k}\right)\right)
$$

\subsection{Convergence and stability}

In this section main results concerning the pathwise convergence and stability of the method are considered. We have the following theorems. 


\subsubsection{Pathwise convergence}

Theorem: Let's suppose that the drift coefficient in equation (2) is Locally Lipschitz and let $\varepsilon>0$. Then, the numerical integrator (11) is almost surely globally convergent and we have that

$$
\sup _{1 \leq n \leq N}\left|x\left(t_{n}\right)-x_{n}\right|=O\left(h^{1-\varepsilon}\right), \quad \text { w.p.1 }
$$

\subsubsection{Mean-Square linear stability}

Stability analysis of numerical integrators concerns the long-term behavior of the numerical map, for fixed step-size $h$. This consists in applying the numerical method to a class of test equations with relevant qualitative features and to analyze the ability of the method to reproduce this feature. One useful measure of stability is the asymptotic Mean-Square stability [5]. For this, consider the equation (1) with $a(x(t))=A x(t)$, and $b_{j}(x(t))=B_{j} x(t)$. In this case, the solution satisfies [5]

$$
\lim _{t \rightarrow \infty}|x(t)|=0 \Longleftrightarrow A+\frac{1}{2} \sum_{j=1}^{m}\left|B_{j}^{2}\right|<0 .
$$

We are interested in numerical methods reproducing this behavior of the continuos SDE. In such a case the method is termed MS-stable. We have the following important result.

Theorem: The numerical integrator (11) is unconditionally MS-stable. That is, it is MS-stable independently of the stepsize $h$.

\section{Implementation issues}

For completeness we now summarize the computational algorithm to compute the approximation $x_{n}$ to the solution of (2) in $t=t_{n}$ :

i) Set an step-size $h$, and consider a partition $t_{0}<t_{1}<\ldots<t_{N}=T$ of $\left[t_{0}, T\right]$

ii) Generate i.i.d $N(0,1)$ random variables $\gamma_{j k}(1 \leq j \leq m, 0 \leq k \leq N-1)$. Compute $I_{j k}$ as in (14) and $I_{j k} \cdot \gamma_{j k}$ as in (15)

iii) Set $z_{0}=x_{0}, \phi\left(t_{0}\right)=1$ and Compute $z_{1}$ in (6)

iv) Repeat from $n=1$ to $n=N-1$ :

(a) Compute $\hat{\phi}\left(t_{n}\right)$ as in (16)

(b) Compute $M$ by using $\hat{\phi}\left(t_{n}\right)$ instead of $\phi\left(t_{n}\right)$ in (13)

(c) Compute $z_{n+1}$ in (12)

v) For $i=1$ to $N$, compute $x_{n}=\hat{\phi}\left(t_{n}\right) z_{n}$ 
Remark 4.1. The numerical computation of the exponential matrix $\exp (M h)$ in (12) can be done in a numerically stable way by using a diagonal Padê approximation with "scaling and squaring" strategy. Nowadays, professional mathematical software, such as MATLAB, provide efficient codes for implementing the Padê algorithm, see e.g., [8] for details.

\section{Conclusions}

In this work we introduced an explicit exponential-based numerical integrator for the computer simulation of SDEs driven by multidimensional linear multiplicative noise. We analyzed the pathwise convergence and MS-stability of the proposed method. The followed strategy essentially consisted in transforming the original SDE into a RDE which in turn was solved by an stable exponential integrator. This approach allows to integrate a wide class of important SDEs in applications, remarkably even those for which standard assumptions on the coefficients are not satisfied. We point out that although -to facilitate the presentation- we focus on scalar SDEs, the method can be adapted without major complications to higher dimensional SDE systems.

\section{References}

[1] L. Arnold, Stochastic Differential Equations: Theory and Applications, John Wiley \& Sons, 1974.

[2] F. Carbonell, J. C. Jimenez, R. J. Biscay and H. de la Cruz, The Local Linearization method for numerical integration of random differential equations, BIT Num. Math., 45:1-14, 2005.

[3] F. Carbonell, R. J. Biscay, J. C. Jiménez and H. de la Cruz, Numerical simulation of nonlinear dynamical systems driven by commutative noise. Journal of Computational Physics, 226 (2): 1219-1233, 2007.

[4] X. Han and P. Kloeden, Random Ordinary Differential Equations and Their Numerical Solution, Springer, 2017.

[5] R. Z. Hasminskii, Stochastic Stability of Differential Equations, Sijthoff Noordhoff, Alphen aan den Rijn, The Netherlands, 1980.

[6] A. Jentzen and P. Kloeden, Taylor Approximations for Stochastic Partial Differential Equations, CBMS-NSF Regional Conference Series in Applied Mathematics, 2011.

[7] P. E. Kloeden and E. Platen, Numerical Solution of Stochastic Differential Equations, Springer-Verlag, Berlin, 1992.

[8] R. B. Sidje, EXPOKIT: software package for computing matrix exponentials, $A M C$ Trans. Math. Software, 24:130-156, 1998. 